\title{
Failure to Recognize the Diagnosis of Cytomegalovirus Colitis in an Immunocompetent Male: Need for Heightened Index of Suspicion
}

\author{
Hugo J.R. Bonatti ${ }^{1,3}$ Jessica L. Hata, ${ }^{2}$ Michael A. Vella, ${ }^{1}$ Chanjuan Shi, \\ William H. Nealon, ${ }^{4}$ and Addison K. May ${ }^{1}$
}

\begin{abstract}
Background: Cytomegalovirus (CMV) disease affects mainly immunosuppressed individuals such as stem cell and solid organ transplant recipients as well as those patients infected with the human immunodeficiency virus (HIV). Cytomegalovirus colitis has been diagnosed increasingly in normal hosts.

Case Presentation: A 65-year-old male with a history of alcohol abuse had been hospitalized for severe bloody diarrhea for three months in an outside hospital; he tested negative for clostridial toxin. He was transferred to our intensive care unit in septic shock and multi-organ failure with bloody diarrhea and a megacolon on computed tomography (CT) scan. Emergency sigmoid colectomy was performed, however, the patient continued to have bloody diarrhea; subsequently, completion sub-total colectomy with ileostomy was performed. On immunohistochemistry a diagnosis of CMV colitis was made; blood CMV polymerase chain reaction (PCR) was positive, the patient tested positive for anti-CMV immunoglobulin (Ig) $G$ but non-IgM antibodies. Ganciclovir was started and the patient's CMV PCR became negative within one week. The patient developed multiple other complications including Aspergillus tracheobronchitis, Pseudomonas aeruginosa sepsis, and Candida krusei pneumonia, but ultimately recovered from the multiple infections. A low natural killer $(\mathrm{NK})$ cell count $(<1 \%)$ was found on immunologic workup; the patient tested negative for HIV.
\end{abstract}

Conclusion: Cytomegalovirus colitis is a rare disease in the non-immunocompromised host but should be considered in patients with pseudomembranous colitis testing negative for clostridial toxin.

C YTOMEGALOVIRUS (CMV) has been associated with a specific fetopathy, pneumonitis in the newborn, and diseases in the immunocompromised host. Before the development of highly active anti-retroviral therapy (HAART), CMV disease was a common complication in human immunodeficiency virus (HIV)-infected individuals. Cytomegalovirus is the most important systemic virus complicating solid organ transplantation. Cytomegalovirus disease manifests most commonly as a mononucleosis-like syndrome, however, the virus may also cause interstitial pneumonitis, retinitis, and meningoencephalitis as well as diseases of the gastrointestinal tract including esophagitis, gastritis, gastric ulcers, enteritis, and colitis. Although CMV colitis is typically observed in severely immunosuppressed patients, increasingly cases of life-threatening CMV colitis have been diagnosed in the normal host [1-12].

Cytomegalovirus plays a pivotal role in inflammatory bowel disease (IBD) by potentially triggering IBD and/or emerging after onset of immunosuppressive therapy including disease modifiers [13-15]. The clinical presentation of CMV colitis ranges from mild diarrhea with minimal mucosal changes to toxic megacolon with pseudomembranes

\footnotetext{
${ }^{1}$ Department of Surgery, ${ }^{2}$ Department of Pathology, Vanderbilt Medical Center, Nashville, Tennessee.

${ }^{3}$ University of Maryland, Community Medical Group Surgical Care, Easton, Maryland.

${ }^{4}$ Yale University New Haven, Connecticut.
}

(C) Hugo J.R. Bonatti et al. 2016; Published by Mary Ann Liebert, Inc. This Open Access article is distributed under the terms of the Creative Commons License (http://creativecommons.org/licenses/by/4.0), which permits unrestricted use, distribution, and reproduction in any medium, provided the original work is properly credited. 
similar to Clostridium difficile-associated colitis (CDAC). Co-infection of CMV together with $C$. difficile has been reported [16]. Once diagnosed, CMV colitis responds to antiviral therapy with ganciclovir [4,7], however, in the nonimmunocompromised host diagnosis is made commonly on colectomy specimens because normal patients with colitis are not tested routinely for CMV. Cytomegalovirus polymerase chain reaction (PCR) is not available universally in many hospitals or not performed routinely on a daily basis but rather once or twice weekly for pooled monitoring of high-risk patients [17]. Antibody testing is usually not conclusive because the seroprevalence of CMV exceeds $60 \%$ and only in rare cases new infection with this virus as determined by presence of CMV-specific IgM antibodies occurs in adults. In the majority of cases, the virus re-emerges from its latent state in endothelial, epithelial, and polymorph nuclear cells after a change in the immune status of patients. This change in the immune competence against CMV may be subtle and difficult to diagnose. Because the most important immune response to the virus is a specific T-cell response, alterations in the T-cell immunity are associated most commonly with CMV re-activation although also critically ill individuals are at risk for this opportunistic infection [18]. We herein report on a middle-aged male who developed pseudomembranous colitis caused by CMV.

\section{Case Presentation}

A 65-year-old male had been treated for several weeks at an outside facility before transfer to our hospital. He was a smoker and had been drinking substantial amounts of alcohol in the past. He had a three-month history of watery and bloody diarrhea, weight loss, malaise, and chronic fatigue. At the outside hospital he received several courses of antibiotics including ciprofloxacin and metronidazole for presumed bacterial diarrhea. He had tested negative for Salmonella, Shigella, Yersinia, Escherichia coli as well as $C$. difficile. No work up for immunologic disorders or for viral pathogens or parasites had been performed. He was malnourished and had lost more than $15 \mathrm{~kg}$ over the past three months; his creatinine was elevated to $1.7 \mathrm{mg} / \mathrm{dL}$ and his bilirubin was $2.5 \mathrm{mg} / \mathrm{dL}$. The patient had been started on coumadin for deep venous

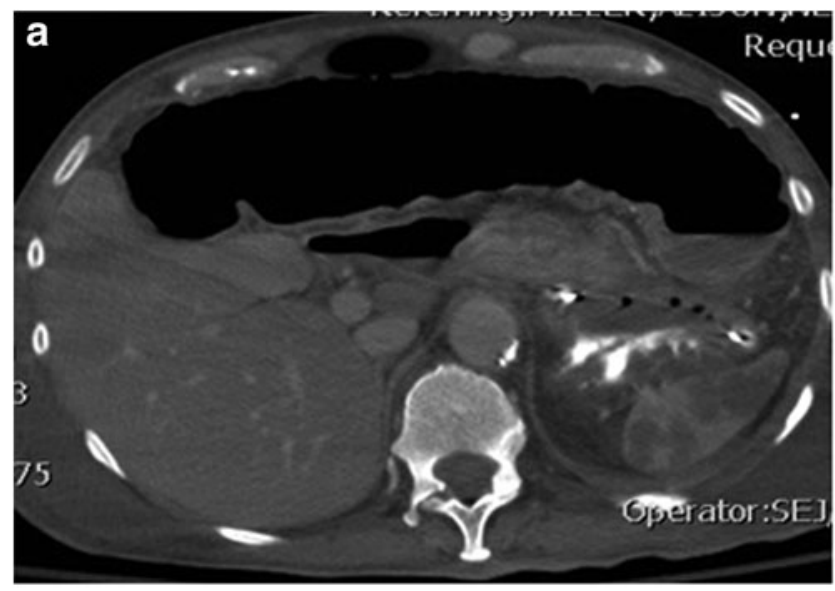

FIG. 1. Abdominal computed tomography (CT) scan showing a distended transverse colon (a) and wall thickening of the sigmoid colon (b).

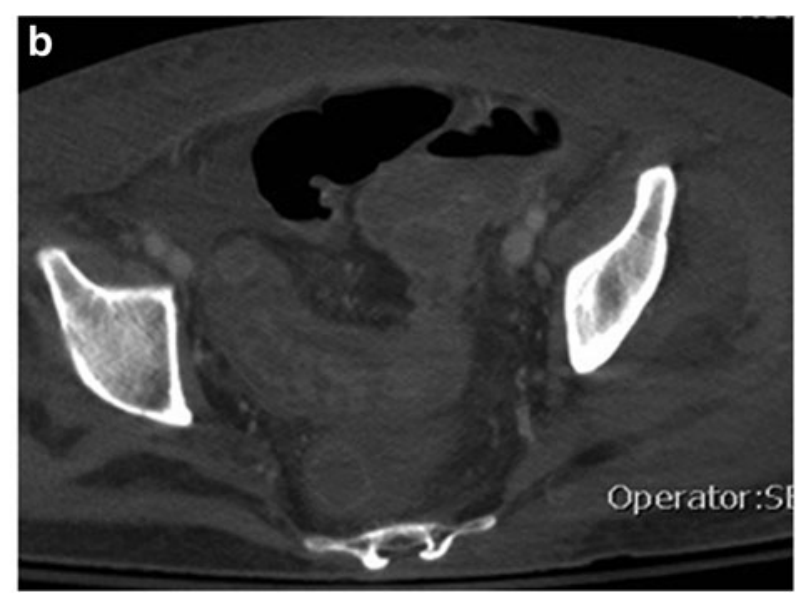

thrombosis at the outside hospital and developed supratherapeutic international normalized ration (INR $>5$ ). Because of respiratory failure he was intubated and transferred to our hospital, where he was admitted to the intensive care unit and fluid resuscitation was begun.

On admission, the patient had a tender distended abdomen. His white blood cell (WBC) count was 17.8 cells per milliliter with a hematocrit of $23 \%$. He was continued on antibiotic therapy including metronidazole, oral vancomycin, and doripenem. Records from the outside hospital showed that the patient had positive sputum cultures for Candida krusei as well as Aspergillus fumigatus and therefore micafungin was started. Clostridium difficile toxin assay was negative as was HIV antibody testing. Computed tomography scan revealed bilateral pneumonia with pleural effusions, a distended colon with significant wall thickening (Fig. 1), and a sub-total infarction of the spleen. The patient had several bloody bowel movements; a colonoscopy seemed to be associated with substantial risk for perforation, and therefore, it was decided to perform emergency laparotomy after correction of the coagulopathy. The left colon was found to be severely inflamed and the sigmoid colon showed maximum pathology and was resected (Fig. 2a); an end descendostomy was created and the rectal stump was stapled. Histopathology showed severe pseudomembranous colitis but a diagnosis of inflammatory bowel disease (IBD) was also discussed. No specific signs of CMV colitis were found on routine hematoxylin and eosin (H\&E) staining and immunohistochemistry was also negative for CMV (Fig. 2b). Cytomegalovirus serology showed positive IgG but negative IgM antibodies. The patient improved slightly after surgery, however, there was still substantial bleeding from the ostomy and the patient required further transfusion of red blood cells. Therefore, a completion sub-total colectomy with end ileostomy was performed. The patient required massive additional blood, platelet, and fresh frozen plasma transfusions, fluid resuscitation, as well as high-dose vasopressor therapy. His course was complicated further by acute cardiac failure and he was started on milrinone $475 \mathrm{mg} / \mathrm{h}$. Because of the undiagnosed cause of the disease, pathology was requested to perform in vitro hybridization of the specimen for potential 

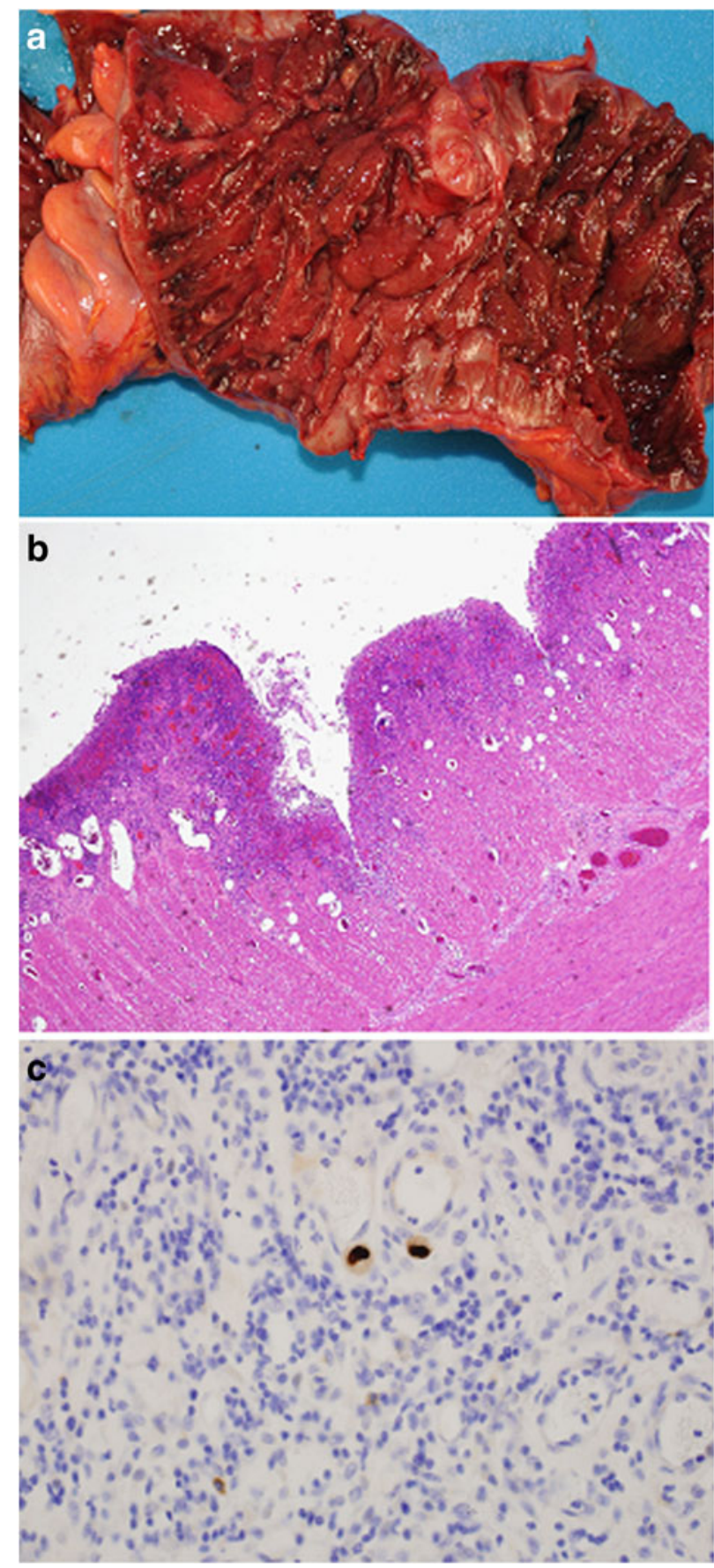

FIG. 2. (a) Macropathology of resected colon shows inflammation and pseudomembranes. (b) Hematoxylin and eosin staining $(40 \times)$ : Inflammatory changes in colonic mucosa. (c) Immunohistochemistry: Intense staining of mucosal cells for cytomegalovirus (CMV).

pathogens. This revealed the diagnosis of CMV colitis (Fig. 2c) although a co-diagnosis of IBD was also discussed. Intravenous ganciclovir at a dose of $10 \mathrm{mg} / \mathrm{kg}$ was started. Also CMV PCR testing was positive with 3,171 copies per milliliter; repeat testing four days and three weeks after initiation of antiviral therapy was negative. Although the patient improved overall, he developed Pseudomonas aeruginosa pneumonia, which was treated with doripenem in combination with gentamicin. Immunologic workup showed that the patient had a low concentration of IgM and IgG and elevated $\operatorname{IgA}$ and $\operatorname{IgE}$ concentrations; in addition his NK cell count was found to be low $(<1 \%)$; his CD4 and CD8 counts were normal. The patient underwent tracheostomy because he could not be weaned off the ventilator; total parenteral nutrition (TPN) was started and later switched to enteral feeding. Gradually his condition improved and the patient could be transferred to a rehabilitation clinic; his tracheostomy was closed and a sacral decubitus was treated surgically. The patient finally recovered and was discharged with a plan for reversal of his ileostomy.

\section{Discussion}

Cytomegalovirus colitis is a rare infection in immunocompetent individuals $[6,11,19]$. However, as this case illustrates, a delay in diagnosis has devastating consequences for the patient. It should also be considered that the low NK cell count in our patient may have been the reason why he re-activated the virus and the infection could not be controlled [20,21]. A de novo infection was excluded by serology and would have been unusual at the age of $50 \mathrm{y}$ in a non-transplant patient. Our patient had had diarrhea for three months and experienced severe weight loss and superinfections with unusual pathogens including atypical fungi [22]. Nevertheless, neither immunologic nor thorough infectious workup was carried out. Clostridium difficileassociated colitis has become a problem in the United States and was suspected despite several negative assays [23]. Negative assays do not exclude $C$. difficile infection, however, one should always consider other enteric infections even if pseudomembranous colitis is observed on colonoscopy [24]. Cytomegalovirus has the potential to mimic CDAC; also co-infection with multiple pathogens both in immunosuppressed and immunocompetent patients has been observed $[3,25]$. Finally, non-infectious causes and a multitude of pathogens such as rotavirus, Mycobacterium tuberculosis, or Cryptosporidia among others may cause megacolon and/or pseudomembranous colitis.

The severe course of our patient's illness with multiple superinfections also outlines the indirect effects that CMV can exhibit [26]. In the transplant population it is well established that in addition to CMV disease, the virus causes paralysis of the immune system giving way to other infections such as other viruses, rare bacteria, and fungi such as Pneumocystis jiroveci, Candida spp., and filamentous fungi. It is difficult to determine whether our patient's NK cell deficiency had been present at onset of disease because baseline values were unavailable [27]. He had a history of alcohol abuse and low NK cell count has been reported in alcoholism [28]. On the other hand, the condition may have been a result of the CMV disease.

Cytomegalovirus PCR can readily establish the diagnosis of CMV disease, however, patients may occasionally test negative on PCR from blood despite gastrointestinal disease [29]. Therefore, colonoscopy with biopsy and specific staining is the test of choice if CMV colitis is suspected. Once diagnosed, treatment with ganciclovir is successful in most patients. Cytomegalovirus colitis should respond to therapy with ganciclovir and only in rare cases surgical intervention 
is necessary [4,7]. This becomes necessary mostly in cases of delayed diagnosis, co-infection with other pathogens, or in immunocompromised individuals who cannot clear the virus despite antiviral therapy.

To summarize, we herein report the case of a patient with undiagnosed CMV colitis, which led to colectomy. Awareness regarding this disease, testing using CMV PCR and/or colonoscopy with biopsy, and specific CMV staining at an early stage followed by treatment with ganciclovir could have resulted in complete remission and could have avoided the permanent disability, the multiple complications, and excessive costs in this patient.

\section{Author Disclosure Statement}

No competing financial interests exist.

\section{References}

1. Begos DG, Rappaport R, Jain D. Cytomegalovirus infection masquerading as an ulcerative colitis flare-up: Case report and review of the literature. Yale J Biol Med 1996; 69:323-328.

2. Carter D, Olchovsky D, Pokroy R, Ezra D. Cytomegalovirusassociated colitis causing diarrhea in an immunocompetent patient. World J Gastroenterol 2006;12:6898-6899.

3. Chau TN, Lau LK, Lee KC, et al. Association of selflimited cytomegalovirus colitis and shigellosis in an immunocompetent patient. Eur J Gastroenterol Hepatol 1996; 8:819-822.

4. Coll PP, Pacala JT, Hamilton CW. Cytomegalovirus colitis in an older woman, successfully treated with ganciclovir. J Fam Pract 1992;34:772-775.

5. Eddleston M, Peacock S, Juniper M, Warrell DA. Severe cytomegalovirus infection in immunocompetent patients. Clin Infect Dis 1997;24:52-56.

6. Galiatsatos P, Shrier I, Lamoureux E, Szilagyi A. Metaanalysis of outcome of cytomegalovirus colitis in immunocompetent hosts. Dig Dis Sci 2005;50:609-616.

7. Jacobson MA. Ganciclovir therapy for severe cytomegalovirus infection in immunocompetent patients. Clin Infect Dis 1997;25:1487-1488.

8. Lin YH, Yeh CJ, Chen YJ, et al. Recurrent cytomegalovirus colitis with megacolon in an immunocompetent elderly man. J Med Virol 2010;82:638-641.

9. Rafailidis PI, Mourtzoukou EG, Varbobitis IC, Falagas ME. Severe cytomegalovirus infection in apparently immunocompetent patients: A systematic review. Virol J 2008; $5: 47$.

10. Hookman P, Barkin JS. Clostridium difficile associated infection, diarrhea and colitis. World J Gastroenterol 2009; 15:1554-1580.

11. Karigane D, Takaya S, Seki Y, et al. Cytomegalovirus enteritis in immunocompetent subjects: A case report and review of the literature. J Infect Chemother 2014;20:325329.

12. Siciliano RF, Castelli JB, Randi BA, et al. Cytomegalovirus colitis in immunocompetent critically ill patients. Int J Infect Dis 2014;20:71-73.

13. Kandiel A, Lashner B. Cytomegalovirus colitis complicating inflammatory bowel disease. Am J Gastroenterol 2006; 101:2857-2865.

14. Omiya M, Matsushita M, Tanaka T, et al. The absence of large ulcer predicts latent cytomegalovirus infection in ulcerative colitis with positive mucosal viral assay. Intern Med 2010; 49:2277-2282.

15. Kishore J, Ghoshal U, Ghoshal UC, et al. Infection with cytomegalovirus in patients with inflammatory bowel disease: Prevalence, clinical significance and outcome. J Med Microbiol 2004;53(Pt 11):1155-1160.

16. Dahman M, Krell R, Brayman K, et al. Simultaneous Clostridium difficile-associated colitis and late-onset intestinal cytomegalovirus disease in a renal transplant recipient. Ann Transplant 2010;22;15:72-76.

17. Halwachs-Baumann G. Recent developments in human cytomegalovirus diagnosis. Expert Rev Anti Infect Ther 2007;5:427-439.

18. Cook $\mathrm{CH}$, Trgovcich J. Cytomegalovirus reactivation in critically ill immunocompetent hosts: A decade of progress and remaining challenges. Antiviral Res 2011;90:151-159.

19. Paparoupa M, Schmidt V, Weckauf H, et al. CMV colitis in immunocompetent patients: 2 cases of a diagnostic challenge. Case Rep Gastrointest Med 2016;2016:4035637.

20. Arase H, Lanier LL. Specific recognition of virus-infected cells by paired NK receptors. Rev Med Virol 2004;14;83-93.

21. Bekiaris V, Gaspal F, McConnell FM, et al. NK cells protect secondary lymphoid tissue from cytomegalovirus via a CD30-dependent mechanism. Eur J Immunol 2009; 39:2800-2808.

22. Bonatti H, Stelzmueller I, Berger N, et al. Infections caused by Candida krusei in five transplant and two surgical patients. Surg Infect 2009;10:265-271.

23. Metzger R, Swenson BR, Bonatti H, et al. Identification of risk factors for the development of clostridium difficileassociated diarrhea following treatment of polymicrobial surgical infections. Ann Surg 2010;251:722-727.

24. Chan KS, Lee WY, Yu WL. Coexisting cytomegalovirus infection in immunocompetent patients with Clostridium difficile colitis. J Microbiol Immunol Infect (2016, in press).

25. Stelzmueller I, Wiesmayr S, Eller M, et al. Enterocolitis due to simultaneous infection with rotavirus and Clostridium difficile in adult and pediatric solid organ transplantation. J Gastrointest Surg 2007;11:911-917.

26. Paya CV. Indirect effects of CMV in the solid organ transplant patient. Transpl Infect Dis 1999;1(Suppl 1):8-12.

27. Pahwa S, Kirkpatrick D, Ching C, et al. Persistent cytomegalovirus infection: Association with profound immunodeficiency and treatment with interferon. Clin Immunol Immunopathol 1983;28:77-89.

28. Redwine L, Dang J, Hall M, Irwin M. Disordered sleep, nocturnal cytokines, and immunity in alcoholics. Psychosom Med 2003;65:75-85.

29. Allice T, Enrietto M, Pittaluga F, et al. Quantitation of cytomegalovirus DNA by real-time polymerase chain reaction in peripheral blood specimens of patients with solid organ transplants: Comparison with end-point PCR and pp65 antigen test. J Med Virol 2006;78:915-922.

Address correspondence to: Dr. Addison K. May Department of Surgery Division of Trauma and Surgical Critical Care Vanderbilt Medical Center 121121 st Avenue South, 404 MAB Nashville, TN 37212

E-mail: addison.may@vanderbilt.edu 
Cite this article as: Bonatti HJR, Hata JL, Vella MA, Shi C, Nealon WH, May A (2016) Failure to recognize the diagnosis of cytomegalovirus colitis in a normal male: need for heightened index of suspicion. Surgical Infections Case Reports 1:1, 156-160, DOI: 10.1089/ crsi.2016.0039 